\title{
Accretion Flow along a Dipolar Field: Application to Intermediate Polars
}

\author{
João Batista Garcia Canalle \\ Physics Institute, State University of Rio de Janeiro, \\ Rua São Francisco Xavier, 524/3023-D, CEP 20559-900, \\ Rio de Janeiro, $R J$, Brazil and \\ Mullard Space Science Laboratory, University College London, \\ Holmbury St Mary, Dorking, Surrey RH5 6NT, United Kingdom \\ Kinwah Wu, Mark Cropper, Gavin Ramsay \\ Mullard Space Science Laboratory, University College London, \\ Holmbury St Mary, Dorking, Surrey RH5 6NT, United Kingdom \\ Curtis J. Saxton \\ Mount Stromlo and Siding Spring Observatory, \\ Research School of Astronomy and Astrophysics, \\ Australian National University, ACT 0200, Australia
}

\begin{abstract}
.
A hydrodynamic formulation for accretion flow channeled by a dipolar magnetic field is constructed using a curvi-linear coordinate system natural to the field structure. We solve the hydrodynamic equations and determine the velocity, density and temperature profiles of the post-shock accretion flow. The results are applied to accretion flows in intermediate polars. We have found that for systems with massive white dwarfs $\left(\sim 1 \mathrm{M}_{\odot}\right)$ the temperature profiles in the flow can differ significantly to those obtained from models in which the accretion column is assumed to be cylindrical.
\end{abstract}

\section{Introduction}

In magnetic cataclysmic variables (MCVs), the accretion flow near the white dwarf is channeled by the magnetic field. The flow is initially supersonic but becomes subsonic near the white-dwarf surface, and an accretion shock is formed. The shock heats the accreting material to temperatures of $\sim 10^{7}-10^{8} \mathrm{~K}$. The shock-heated material is ionized and emits bremsstrahlung X-rays and cyclotron optical/IR radiation (Lamb \& Masters 1979). The height of the shock above the white-dwarf surface is determined by the cooling processes. It had been believed that the height is small $\left(\sim 10^{5}-10^{6} \mathrm{~cm}\right.$, see Wickramasinghe \& Meggitt 1985), and therefore the shock-heated emission region could be treated as a stack of thin pancake-like plasmas on the white-dwarf surface. More recent studies showed that for some MCV parameters the shock height can be comparable to the 

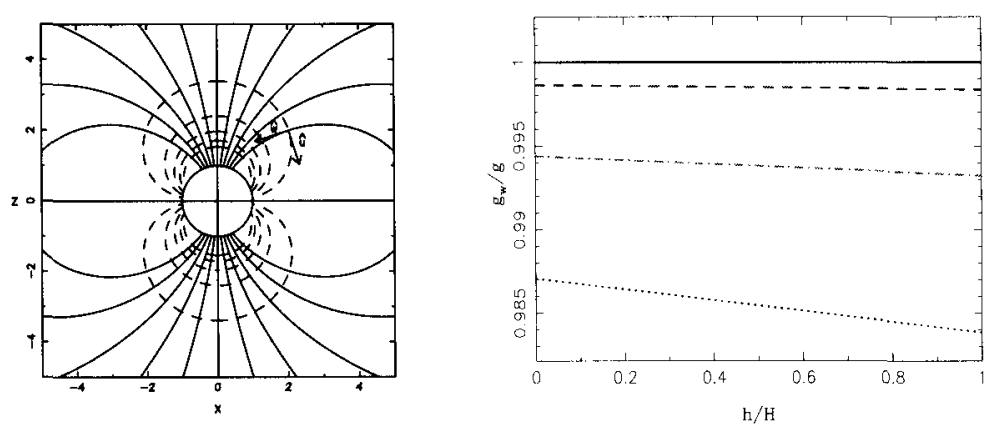

Figure 1. (Left) The $(u, w)$ plane of the curvi-linear coordinate system used in the formulation. Solid curves represent dipole magnetic field lines, and dashed curves represent field equi-potential surfaces. (Right) The $w$ component of the gravity force, $g_{w}$, as a function of height $h$, for field lines with different foot points. The force component is normalized to that of the field line along the polar axis, $g$, and the height is normalized to the shock height $H$. The lines (from top to bottom) correspond to the gravity force along the field lines with foot points at magnetic co-latitude $\theta=0^{\circ}, 6^{\circ}, 12^{\circ}$ and $18^{\circ}$ respectively.

white-dwarf radius when the effects of gravity along the flow are considered (see e.g. Cropper et al. 1999). The latter studies predicted X-ray spectra in good agreement with the grating spectra of MCVs obtained by XMM-Newton and Chandra (see e.g. Cropper et al. 2002).

Most existing hydrodynamic calculations of the post-shock accretion flow in MCVs assume a simple geometry, such as a semi-infinite slab or a cylinder (Aizu 1973; Chavelier \& Imamura 1982; Wu, Chanmugam \& Shaviv 1994; Cropper, Ramsay \& Wu 1998). These simple geometries are good approximations, provided that the shock height is small and the flow is azimuthally symmetric and aligned close to the polar axis. For accretion flows in intermediate polars, these two conditions are not always satisfied, and hence, the effect of the magnetic field geometry needs to be investigated in order to obtain accurate velocity and temperature profiles of the accretion flow.

Here, we present a hydrodynamic formulation in terms of a curvi-linear coordinate system natural to channeled flows in dipole magnetic fields. We solve the hydrodynamic equations and determine the velocity, density and temperature structures of the post-shock region. The results are compared with those of Cropper et al. (1999), in which an azimuthal, cylindrical accretion column is assumed.

\section{Formulation}

We assume the accreting material is an ideal gas, with a pressure $P$, density $\rho$ and temperature $T$, related by $P=\rho k_{\mathrm{B}} T / \mu m_{\mathrm{H}}$, where $k_{\mathrm{B}}$ is the Boltzmann 
constant, $m_{\mathrm{H}}$ is the hydrogen mass, and $\mu$ is the mean molecular weight. In stationary accretion, the hydrodynamic equations governing the flow are

$$
\begin{aligned}
\nabla \cdot(\rho \mathbf{v}) & =0, \\
(\mathbf{v} \cdot \nabla) \mathbf{v}+\frac{1}{\rho} \nabla P & =\mathbf{g}, \\
(\mathbf{v} \cdot \nabla) P-\frac{\gamma P}{\rho}(\mathbf{v} \cdot \nabla) \rho & =-(\gamma-1) \Lambda,
\end{aligned}
$$

where $\mathbf{v}$ is the velocity of the flow, $\gamma$ is the adiabatic index of the gas, $\mathbf{g}$ is the gravitational acceleration, and $\Lambda$ is the effective cooling function. (See Wu (2000) and references therein for the hydrodynamic formulation of accretion flow in MCVs.)

We consider a coordinate system $(u, w, \varphi)$, in which the orthogonal unit vectors $\hat{u}, \hat{w}$ and $\hat{\varphi}$ are defined as follows: $\hat{u}$ is on the equi-potential surface of the field at a fixed azimuthal angle, $\hat{w}$ is along the magnetic field line, and $\hat{\varphi}$ is the same as that for the azimuthal coordinate in the spherical coordinate system (see Fig. 1, left panel). The metrics of the coordinate system, $h_{1}, h_{2}$ and $h_{3}$, are given by

$$
\begin{aligned}
& h_{1}=\sqrt{\left(\frac{\partial x}{\partial u}\right)^{2}+\left(\frac{\partial y}{\partial u}\right)^{2}+\left(\frac{\partial z}{\partial u}\right)^{2}} \\
& h_{2}=\sqrt{\left(\frac{\partial x}{\partial w}\right)^{2}+\left(\frac{\partial y}{\partial w}\right)^{2}+\left(\frac{\partial z}{\partial w}\right)^{2}} \\
& h_{3}=\sqrt{\left(\frac{\partial x}{\partial \varphi}\right)^{2}+\left(\frac{\partial y}{\partial \varphi}\right)^{2}+\left(\frac{\partial z}{\partial \varphi}\right)^{2}}
\end{aligned}
$$

where $(x, y, z)$ is the Cartesian coordinate system. We may then express the hydrodynamic equations in terms of $(u, w, \varphi)$.

We integrate the mass continuity equation directly and obtain $h_{1} h_{3} \rho v=$ $C$, where $C$ is a constant. Substituting this into the other two hydrodynamic equations yields

$$
\begin{aligned}
\frac{\partial \xi}{\partial w} & =-h_{2}\left[\frac{g_{w}}{v}-\frac{\mathcal{H}}{h_{2}}(\xi-v)\right] \\
\frac{\partial v}{\partial w} & =-\left(\frac{h_{2}}{\gamma(\xi-v)-v}\right)\left[\frac{\gamma-1}{C} h_{1} h_{3} \Lambda+\frac{\gamma \mathcal{H}}{h_{2}}(\xi-v) v-g_{w}\right] .
\end{aligned}
$$

Here, $\xi \equiv v+(P / \rho v), g_{w}$ is the gravitational acceleration projected on $\hat{w}$ (Fig. 1 , right panel), and the function $\mathcal{H}(u, w)=\partial \ln \left(h_{1} h_{3}\right) / \partial w$, describes the change of cross-section area of the flow-flux tube.

Our cooling function $\Lambda$ consists of two parts, one for bremsstrahlung cooling and another for cyclotron cooling. We adopt the composite cooling function of $\mathrm{Wu}$ (1994) and Wu et al. (1994) (see also Cropper et al. 1998). As cyclotron 


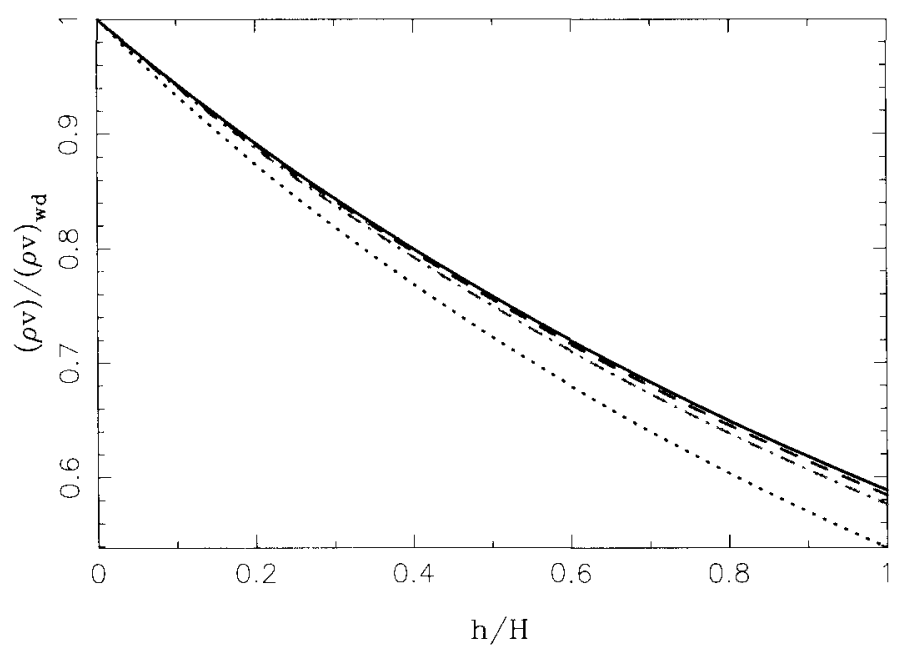

Figure 2. The quantity $\rho v$ as a function height along the field lines, normalized to the values, $(\rho v)_{\mathrm{wd}}$, at the white-dwarf surface. From top to bottom, the foot points of the field lines are at magnetic co-latitudes $\theta=0^{\circ}, 6^{\circ}, 12^{\circ}$ and $18^{\circ}$ respectively.

cooling is practically unimportant in intermediate polars, we simply set the efficiency parameter $\epsilon_{\mathrm{s}}=0$, i.e. $\Lambda=A \rho^{2}(P / \rho)^{1 / 2}$, where the constant $A=$ $3.9 \times 10^{16}$ in c.g.s. units. We assume a cold stationary wall for the lower boundary condition as in Chevalier \& Imamura (1982) and Wu et al. (1994). For the upper boundary condition a strong adiabatic shock is considered, but with this coordinate system its location must be determined self-consistently. Detailed treatment of the upper boundary condition will be presented in Canalle et al. (2003). With the cooling function and the boundary conditions defined, we can now integrate the hydrodynamic equations along the field lines numerically and obtain the velocity, density and temperature profiles of the flow.

\section{Results and Discussion}

In all calculations, unless otherwise stated, the white-dwarf mass $M_{\mathrm{wd}}=1 \mathrm{M}_{\odot}$ and the specific mass accretion rate $\dot{m}=2.0 \mathrm{~g} \mathrm{~cm}^{-2} \mathrm{~s}^{-1}$. The white-dwarf radius, $R_{\mathrm{wd}}$, is determined by the Nauenberg (1972) mass-radius relation.

For comparison we also calculate the velocity, temperature and density profiles of a flow with an azimuthal symmetric cylindrical accretion column using the formulation in Cropper et al. (1999). The two main differences in the two formulations are that for flows channeled strictly by dipolar magnetic fields the cross section of the flow-flux tube decreases with distance to the white-dwarf surface and that the gravitational acceleration varies in strength and direction along the flow. As shown in Figure 1 (right panel), for the parameters assumed the gravitational accelerations are smaller for the flows along field lines with foot point co-latitudes $\theta$ farther away from the pole. With the parameters as- 


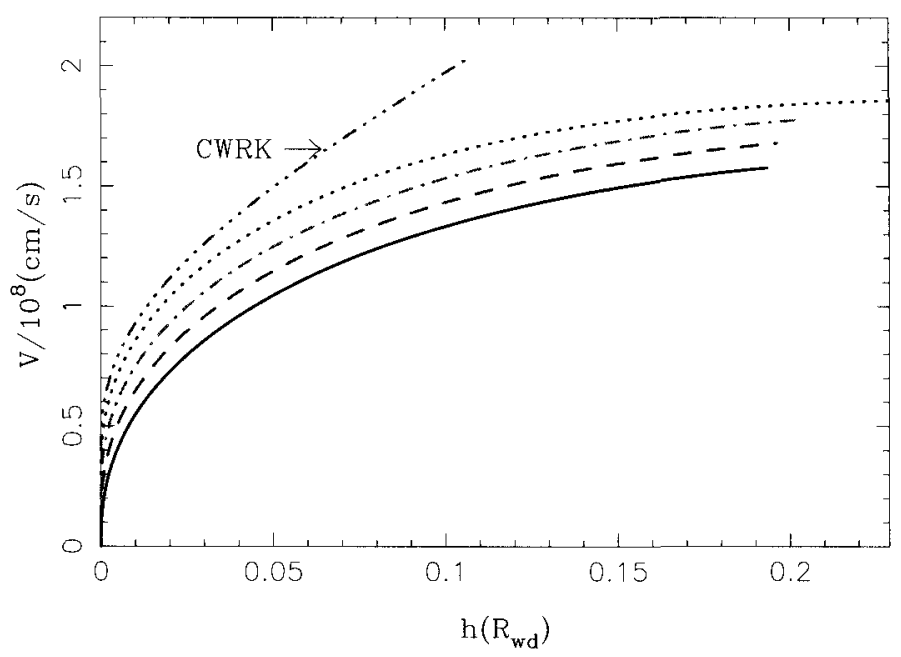

Figure 3. The velocity profiles of the post-shock accretion flow. The solid, dashed, dash-dotted and dotted curves correspond to flows along field lines with foot points at magnetic co-latitudes $\theta=0^{\circ}, 6^{\circ}, 12^{\circ}$ and $18^{\circ}$, respectively The dash-dot-dot-dotted curve (labelled CWRK) corresponds to the velocity profile obtained from the calculation following Cropper et al. (1999). The curves are terminated at the heights where the shocks are located. The velocities of the curves are shifted progressively upward each by $0.1 \times 10^{8} \mathrm{~cm} \mathrm{~s}^{-1}$ to amplify their differences.

sumed in Figure 1, the value of $g_{w} /|\mathbf{g}|$ at the shock is about 0.985 for $\theta=18^{\circ}$ : the value can be much smaller for larger $\theta$ and also for parameters which yield shock heights significantly above the white-dwarf surface. Figure 2 shows the $\rho v$ profiles for various $\theta$. For small $\theta$, the profiles are similar, but when $\theta$ is substantially large the deviation becomes significant: compare the profiles of $\theta=12^{\circ}$ and $18^{\circ}$ with those of smaller $\theta$.

Figure 3 shows the velocity profiles of the flows. The shape of the profiles for the flows are quite similar regardless of the differences between the values of $\theta$. However, the shock heights are different, and hence the shock temperatures are different accordingly. Interestingly, the profiles are quite different to those of the cylindrical accretion column (Cropper et al. 1999). In particular, the latter yield a smaller shock height, thus a hotter and more compact post-shock emission region. This will affect the properties of the optical/IR cyclotron radiation as they are emitted mainly from the hotter part of post-shock region. The effects on the bremsstrahlung X-ray continuum may be less affected as it is emitted from the cooler bottom of the post-shock region.

Figure 4 shows the temperature profiles for systems with various whitedwarf masses. Both the flows in cylindrical columns and flows confined by dipolar fields show that the shock temperatures increases with the white-dwarf mass. For white-dwarf with masse $\lesssim 0.7 \mathrm{M}_{\odot}$, the profiles for the two cases are similar, with the model with cylindrical columns giving slightly higher temperatures. 


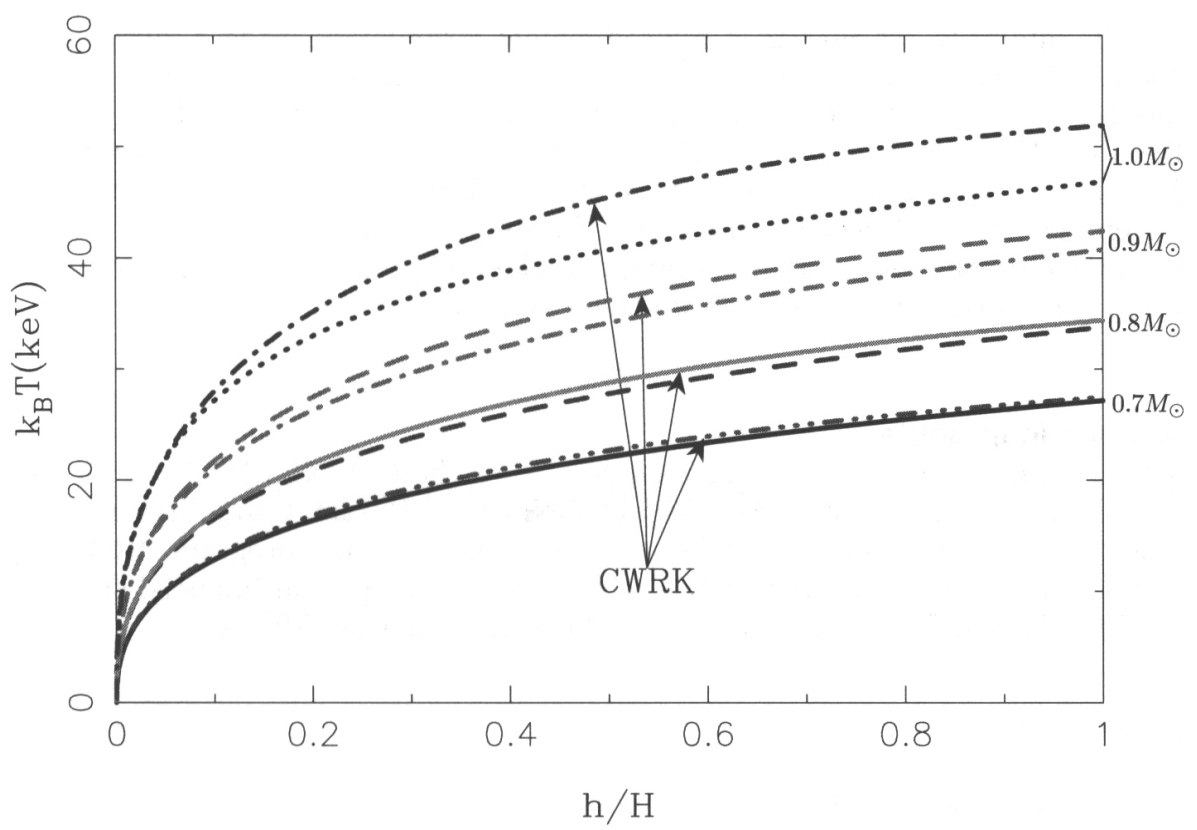

Figure 4. Temperature profiles of flows along a dipolar field in comparison with flows assuming a cylindrical accretion column (labeled CWRK, Cropper et al. 1999) for white-dwarf masses 1.0, 0.9, 0.8 and $0.7 \mathrm{M}_{\odot}$ (curve pairs from top to bottom). For the flows along a dipolar field, the foot point of the field line is fixed at the magnetic co-latitude $\theta=18^{\circ}$. In all cases, the mass accretion rate $\dot{m}=2.0 \mathrm{~g} \mathrm{~cm}^{-2} \mathrm{~s}^{-1}$.

However, when the white-dwarf mass is sufficiently large $\left(\sim 1.0 \mathrm{M}_{\odot}\right)$, the differences between the two cases becomes obvious. Also, the model with cylindrical columns tends to overpredict the gas temperatures. The difference between the two cases can be explained qualitatively as follows. Larger white-dwarf masses result in higher shock temperatures and hence larger shock heights above the white-dwarf surface. When the shock height becomes too large, the cylindrical accretion column is not a very good approximation, and the variation in the gravitational acceleration along the flow becomes significant. The field geometry has stronger effects on the accretion onto massive white dwarfs than low-mass white dwarfs. Therefore, when modelling the $\mathrm{X}$-rays and optical/IR radiations from the shock-heated accreting gas in the systems with massive white dwarfs, one must consider the effects due to the field geometry.

These finding may explain the finding of Cropper et al (1999) and Ramsay (2000) who found that the mass of the white dwarf in MCVs were biased towards higher masses compared to isolated white dwarfs when they fitted X-ray spectra with the cylindrical formulation. Work is currently in progress to implement the affect of the field geometry and determine how it will affect the resulting white dwarf masses. 
In summary, we present a hydrodynamic formulation using a curvi-linear coordinate system defined by the dipole field configuration. We use this formulation to calculate the velocity, density and temperature profiles of the accretion flows in MCVs. We have shown that effects of field geometry are important for MCVs with high-mass white dwarfs. Our formulation improves the calculations of temperature and density structures of post-shock emission region in MCVs, in particular, the intermediate polars, and thereby predict more accurately the $\mathrm{X}$-ray and optical/IR emission from these systems.

\section{Acknowledgments}

JBGC acknowledges the financial support from the Conselho Nacional de Desenvolvimento Cientifico e Tecnologico (CNPq) and to the State University of $\mathrm{R}$ io de Janeiro (UERJ) for the leave of one year inside the program PROCAD to take part in this research. JBGC also acknowledge partial financial support received from IAU to take part in the IAU Colloquium on MCVs.

\section{References}

Aizu, K. 1973, Prog. Theor. Phys., 49, 1184

Canalle, J.B.G., et al. 2003, MNRAS, to be submitted

Chevalier, R.A., \& Imamura, J. N. 1982, ApJ, 261, 543

Cropper, M., Ramsay, G., \& Wu, K. 1998, MNRAS, 293, 222

Cropper, M., Wu, K., Ramsay, G., \& Kocabiyik, A. 1999, MNRAS, 306, 684

Cropper, M., Ramsay, G., Hellier, C., Mukai, K., Mauche, C., \& Pandel, D., 2002, Trans. Phil. Trans. R. Soc. Lon. A, 360, 1951

Lamb, D.Q., \& Masters, A. R. 1979, ApJ, 234, L117

Nauenberg, M. 1972, ApJ, 175, 417

Ramsay, G., 2000, MNRAS, 314, 403

Wickramasinghe, D. T., \& Meggitt, S. M. A. 1985, MNRAS, 214, 605

Wu, K. 1994, Proc. Astron. Soc. Australia, 11, 61

Wu, K. 2000, Space Sci. Rev., 93, 611

Wu, K., Chanmugam, G., \& Shaviv, G. 1994, ApJ, 426, 664 